\title{
The role of action control in implementing intentions during the first weeks of behaviour change
}

\author{
Falko F. Sniehotta ${ }^{1 *}$, Gabriel Nagy ${ }^{2}$, Urte Scholz ${ }^{3}$ \\ and Ralf Schwarzer ${ }^{3}$ \\ 'University of Aberdeen, UK \\ ${ }^{2}$ Max Planck Institute for Human Development, Berlin, Germany \\ ${ }^{3}$ Freie Universität Berlin, Germany
}

\begin{abstract}
Prevailing social cognition models consider behavioural intentions as immediate precursors of actions. This view ignores the role of more proximal self-regulatory processes, such as action control. The latter emerges after an intention has been formed and is supposed to maintain the level of intentions over time and to translate them into action. Three facets of action control were examined in terms of their predictive power for changes in intentions and for physical exercise: (a) awareness of standards, (b) self-monitoring, and (c) self-regulatory effort. A parsimonious 6-item instrument was administered to 122 cardiac patients at six weekly measurement points in time following rehabilitation. A distinction was made between the level of action control and the degree of change in action control, applying a latent growth model. While awareness of standards remained stable, the other two facets exhibited a linear change over the six-week period. Level and change were distinct predictors of physical exercise and changes in intentions. These findings emphasize the importance of selfregulatory mechanisms in the first weeks of trying to overcome a sedentary lifestyle. Action control may be a promising construct to narrow the intention-behaviour gap.
\end{abstract}

Relationships between decision making and human action are frequently studied in social psychology. Social cognition models were proposed specifying beliefs and attitudes as predictors of behavioural intentions, which in turn are supposed to influence subsequent behaviour (Sutton, 2001). The theory of reasoned action (TRA; Fishbein \& Ajzen, 1975) represents this line of thinking. The TRA has been applied across a wide range of social behaviours with some success in predicting (Armitage \& Conner, 2001; Sheeran, 2002). However, people often fail to act on their intentions

* Correspondence should be addressed to Dr Falko F. Sniehotta, University of Aberdeen, School of Psychology, College of Life Sciences and Medicine, King's College, William Guild Building, Aberdeen AB24 2UB, UK (e-mail: f.sniehotto@abdn.ac.uk). 
(Orbell \& Sheeran, 1998) or to maintain difficult intentions over time (Einstein, McDaniel, Willifort, Pagan, \& Dismukes, 2003; Kuhl \& Goschke, 1994).

The theory of planned behaviour (TPB) adds perceived behavioural control (PBC) to the TRA (Ajzen, 1991). PBC, which refers to the perceived extent of control that a person has over performing behaviour, is specified as an additional predictor of intentions, along with attitudes and subjective norm. Moreover, PBC is also considered to contribute directly to the prediction of behaviour. This improves the overall predictive power of the model as compared with the TRA (Armitage \& Conner, 2001; Sheeran, 2002; Sutton, 2001). The strength of this direct effect depends on the accuracy with which PBC reflects actual control (Ajzen, 1991; Sheeran, Trafimow, \& Armitage, 2003). Social cognition models such as the TRA or TPB elaborate constructs at the pre-intentional phase of the change process. However, these models make no further elaboration on the postintentional phase. Post-intentional constructs are more proximal to the actual behaviour. Adding post-intentional constructs has therefore the potential to improve of the predictive power of Social Cognition Models (Abraham, Sheeran, \& Johnston, 1998; Gollwitzer, 1999; Lippke, Ziegelmann, \& Schwarzer, 2004, Sniehotta, Scholz, \& Schwarzer, 2005 ). Thus, identifying psychological constructs and processes that are located between intentions and behaviour seems to be a promising approach to bridge the gap between intention and behaviour. In other words, it is suggested to focus research on the mechanisms that operate after people have developed a behavioural intention. These are self-regulatory mechanisms, such as strategic planning; taking initiative, overcoming barriers, monitoring initial steps, preventing relapse and recovering from lapses.

The present study, therefore, addresses such self-regulatory mechanisms. It examines the role of action control (Carver \& Scheier, 1998; Ford, 1987; Karoly, 1993; Kuhl \& Fuhrmann, 1998, Sniehotta, Scholz, \& Schwarzer, 2005), in particular, inter-individual differences in the levels and in the changes within the first weeks of adopting a new behaviour. It gauges their predictive power in explaining intentions and behaviours.

\section{Proximal action regulation: Negative feedback control}

Negative feedback control (Carver \& Scheier, 1998; Ford, 1987; Karoly, 1993, Maes \& Karoly, 2005) aims at comparing incoming information to individual standards and at reducing possible differences. Thus, it is the most proximal process in action control. Feedback loops serve as basic units in cybernetic control models (Carver \& Scheier, 1998). They can be positive (aiming at increasing discrepancies between input and standard, e.g. avoidance of dangerous activities) or negative (aiming at reducing discrepancies between input and standard, e.g. adhering to a prescribed training programme). A feedback loop consists of at least four elements: (a) an input value gathered by an information collection function; (b) a standard value; (c) a comparator analysing differences between input and standard; and (d) an output function aiming at reducing differences between input and standard (Carver \& Scheier, 1998). For behavioural self-regulation, three cognitive processes can be derived, building upon these models: (a) awareness of standards for action; (b) self-monitoring; and (c) discrepancy-reducing self-regulatory effort (Karoly, 1993).

Once a goal has been set, standards or criteria for action need to be specified (e.g. 'I want to exercise three times a week at the gym'). Moreover, these standards must be called up from the memory to be aware during the course of action (Ford, 1987). This is an active process. Without awareness of standards, the ground for self-guided behaviour is missing (Baumeister, Heatherton, \& Tice, 1994). 
Self-monitoring (Bandura, 1997; Kanfer \& Karoly, 1972) is essential in action control as long as a behaviour has not become a habit. To control their behaviour, individuals must monitor their actions to evaluate whether they are on track and if further selfregulatory effort is needed. Self-monitoring brings up the input information about one's behaviour (e.g. 'I have not exercised for 5 days') to allow comparisons with one's standards. Therefore, lack of awareness in standards or lack in self-monitoring may lead to self-regulation failure (Baumeister et al., 1994). For the purpose of this paper, the process of comparison (Bandura \& Cervone, 1983) is implied in awareness of standards and self-monitoring.

If discrepancies between one's action and standards are detected, discrepancyreducing means must be applied (Bandura \& Cervone, 1983). Previously formed action plans, specifying where, when and how to act (Gollwitzer, 1999) or coping plans, specifying how to cope with barriers and difficulties (Sniehotta, Schwarzer, Scholz, \& Schüz, 2005) can provide such means. Nevertheless, even if precise responses are at hand, self-regulatory effort is required to adhere to one's intentions and plans and to administer action control.

Action control helps to maintain intentions that are difficult to implement (Kuhl \& Goschke, 1994; Kuhl \& Kazén-Saad, 1989). It has been shown that individuals holding unstable intentions are less likely to act on their intentions (Bagozzi \& Yi, 1989; Conner, Sheeran, Norman, \& Armitage, 2000). Maintaining intentions is a major process in action control (Kuhl \& Fuhrmann, 1998). There are at least two mechanisms by which action control contributes to the maintenance of intentions - a mnemonic one and a motivational one. Awareness of standards implies an active prospective memory of the specific intention to be acted upon (McDaniel \& Einstein, 2000), keeping it more salient than competing intentions that require less action control. Discrepancies between perceived behavioural control and the actual amount of control were found to weaken the intention-behaviour relationship (Sheeran et al, , 2003). The experience of failure and of a lack of accuracy in one's PBC might lead to a readjustment of beliefs and intentions. The experience of control, in turn, should facilitate the intention maintenance (Bandura, 1997; Carver \& Scheier, 1998).

Awareness of standards, self-monitoring and self-regulatory effort are conceptually distinct facets of action control. Self-regulation failures can occur in any of these processes (Baumeister $e t$ al., 1994). The interplay of awareness of standards, selfmonitoring and self-regulatory effort can hardly be disentangled in applied settings and correlational designs. However, these three facets of action control may partly mediate the influence of other predictors, such as intentions, action planning and selfefficacy (Sniehotta, Scholz, \& Schwarzer, 2005).

\section{Change in action control}

After a behavioural intention is formed, action-controlling routines must be initiated, orchestrated and applied. Successful patterns are likely to be reinforced by the experience of control (Bandura \& Cervone, 1983). Temporary lapses in action control can activate ruminations that direct the attention away from the planned action and lead to a further loss of control (Baumeister et al., 1994; Kuhl \& Goschke, 1994). Many attempts for lifestyle change fail within the first weeks. The first weeks of an attempt to adopt a new behaviour may thus be characterized by changes in action control. These changes in action control might contribute to the prediction of behaviour. 
In sum, there is compelling evidence that behavioural intentions are crucial for the self-regulation of behaviour. However, only if people maintain their intentions over time and actively control their ongoing behaviour will they attain their goals. Action control is assumed to facilitate intention maintenance and behaviour enactment. Action control can be understood as negative feedback control and characterized by three processes: self-monitoring, awareness of standards and regulatory effort in reducing discrepancies between standards and actual behaviour. It is assumed that levels and changes of these action control facets are predictive of behaviour and mediate the intention-behaviour relationship.

\section{The present study}

To test the predictive power of action control processes in the first weeks of an intended behaviour change, the present study investigates physical exercise in post-rehabilitation patients with coronary heart disease (CHD). Here, patients are usually highly motivated to engage in physical exercise. Since the training programme in the rehabilitation centre is externally controlled and scheduled by the health care providers, the best startingpoint for the study of self-regulation is on the day of discharge from the formal rehabilitation setting. After discharge, it is expected that the individual's amount and direction of change in action control is related to subsequent physical exercise and changes in behavioural intentions. It was hypothesized that initial levels and changes in action control facets will (a) be predictive of physical exercise over and above behavioural intentions and will mediate the effect of behavioural intentions on physical exercise, and that level and changes in action control will (b) be predictive of changes in behavioural intentions. To test these hypotheses, it is crucial to investigate whether the action control facets show longitudinal distinctiveness that allows for further analyses of longitudinal interrelations between them, and whether the three facets display a type of change that allows the notion of inter-individual differences in intra-individual change (latent growth model).

\section{Method}

\section{Sample and procedure}

Participants of the present study were $122 \mathrm{CHD}$ in-patients with the medical recommendation for regular exercise. Their mean age was 59 years $(S D=9.4)$. The majority of participants $(101=82.8 \%)$ were men. Participants completed an initial questionnaire within the rehabilitation (Time 1) and a follow-up questionnaire two months after discharge (Time 2). Additionally, they received six weekly questionnaires assessing action control.

\section{Measures}

Behavioural intentions were measured at Time $1(\alpha=.82 ; M=3.46 ; S D=.54)$ and Time $2(\alpha=.86 ; M=3.49 ; S D=.57 ; 6.6 \%$ missing data) by 6 items validated in a sample of cardiac rehabilitation patients (Sniehotta et al., 2005). The stem ' $I$ intend to. .' was followed by six recommended activities, for example, '. . be physically active regularly for a minimum of 30 minutes at least three times a week' or '. . . elevate my heart rate to the levels recommended in the rehab for at least 30 minutes three times a week' (4-point scale; strongly disagree to strongly agree). 
The criterion behaviour was physical exercise. To assess the strenuous exercise at Time 2, participants were asked in a first step to indicate how many sessions they practised, and how much time per session on average they engaged in (a) vigorous exercise (e.g. swimming), (b) fitness activities (e.g. gymnastics), (c) game sports, such as volleyball or tennis, (d) moderate exercise to train muscle strength and (e) other prescribed exercises. In a second step, they indicated how many of these activities equalled the strain of their exercise programme in the rehabilitation centre. This last information resulted in an average recommended strenuous exercise of 129.03 minutes with a standard deviation of 121.2 minutes. Eleven persons (9\%) had missing values.

The three facets of action control were assessed in the weekly questionnaires using 2 items for each facet: awareness of standards, self-monitoring, and self-regulatory effort (Sniehotta et al., 2005). All items were introduced by the stem 'during the last week, I have. .' with items following to assess awareness of standards (Items a and b), selfmonitoring (Items $\mathrm{c}$ and $\mathrm{d}$ ) and self-regulatory effort (Items $\mathrm{e}$ and $\mathrm{f}$ ): (a) ' . . often had my exercise intention on my mind,' (b) ' . . always been aware of my prescribed training programme', (c) '. . consistently monitored myself whether I exercise frequently enough', (d) '. . taken care to train for at least 30 minutes at the recommended strain level per unit', (e) '. . really tried hard to exercise regularly' and (f) '. . done my best to act in accordance with my standards' (4-point scale; strongly disagree to strongly agree). Internal consistencies, means, standard deviations, percentage of missing data, skewness and kurtosis of these measures for all six weekly questionnaires are shown in Table 1.

\section{Data analysis}

Initial level and change in action control facets were supposed to predict physical exercise and changes in intentions. Before addressing this main hypothesis, two technical assumptions for the final analyses were tested. The first refers to the longitudinal factor structure of the three action control facets, and the second to the type of change within the action control facets.

Longitudinal factor structure of the action control focets

Awareness of standards, self-monitoring and self-regulatory effort are defined as facets of one over-arching action control construct. They are expected to be highly intercorrelated, but nevertheless separable. The distinctiveness of the three action control facets may be defined with respect to different time frames. From a trait perspective, relatively stable inter-individual differences may exhibit a three-factor structure, defined by the action control facets. From a state perspective, in contrast, individual's week-to-week fluctuation in action control may reflect the same three-factor structure. Thus, both, a between- and a within-subjects level indicate whether it is useful to investigate stability and change for each facet separately.

Investigating the factor structure on both levels is a critical aspect of the present study. In order to investigate the longitudinal factor structure of the action control measures on a between- and a within-person level, multi-level confirmatory factor analytic models (MCFA; Hox, 2002) were fitted to the data. Longitudinal data reflect a multi-level structure because measurement occasions are nested within individuals. The solutions determined for the different levels indicate the dimensionality of stable interindividual differences and week-to-week fluctuations in action control, respectively. 
Table I. Summary statistics, reliability estimates (Cronbach's $\alpha$ ), percentage of missing data (\% miss) and skewness $(S)$ and kurtosis $(K)$ for the weekly measured action control facets

\begin{tabular}{|c|c|c|c|c|c|c|c|c|c|c|c|c|c|c|c|}
\hline & \multicolumn{5}{|c|}{ Awareness of standards } & \multicolumn{5}{|c|}{ Self-monitoring } & \multicolumn{5}{|c|}{ Self-regulatory effort } \\
\hline & $M(S D)$ & $\alpha$ & $\%$ miss & $s$ & K & $M(S D)$ & $\alpha$ & $\%$ miss & 5 & K & $M(S D)$ & $\alpha$ & $\%$ miss & $s$ & $k$ \\
\hline Time 1 & $3.33(0.69)$ & .73 & 5.7 & -1.12 & .97 & $3.21(0.81)$ & .68 & 6.6 & -.81 & -.30 & $3.30(0.77)$ & .80 & 5.7 & -1.10 & .88 \\
\hline Time 2 & $3.23(0.74)$ & .77 & 7.4 & -.90 & .69 & $3.26(0.79)$ & .71 & 4.9 & -.95 & .30 & $3.35(0.76)$ & .80 & 6.6 & -1.29 & 1.43 \\
\hline Time 3 & $3.40(0.65)$ & .82 & 7.4 & -1.12 & 1.40 & $3.34(0.72)$ & .65 & 4.9 & -1.10 & 1.00 & $3.43(0.71)$ & .94 & 7.4 & -1.39 & 2.21 \\
\hline Time 4 & $3.29(0.78)$ & .82 & 6.6 & -.93 & .36 & $3.33(0.70)$ & .60 & 4.9 & -.80 & .06 & $3.37(0.71)$ & .82 & 7.4 & -1.10 & 1.31 \\
\hline Time 5 & $3.32(0.74)$ & .90 & 9.8 & -.85 & .26 & $3.23(0.83)$ & .68 & 8.2 & -.98 & .32 & $3.38(0.79)$ & .89 & 9.8 & -1.24 & 1.09 \\
\hline Time 6 & $3.40(0.71)$ & .85 & 9.0 & -.96 & .36 & $3.35(0.76)$ & .70 & 7.4 & -1.29 & 1.59 & $3.34(0.81)$ & .89 & 8.2 & -1.27 & 1.38 \\
\hline
\end{tabular}


It was expected that the measures of action control exhibit a three-dimensional structure on both the between- and within-individual level, representing awareness of standards, self-monitoring and self-regulatory effort.

Type of change in the facets of action control

In order to test initial level and change of action control facets for their predictive power, it had to be checked whether the data were suitable for such an analysis. That is, it has been investigated whether individuals' change in action control followed a pattern that allows the notion of inter-individual differences in the amount of intra-individual change. To do so, it is essential that individuals display the same type of change across occasions (e.g. linear). The applicability of three models, a 'latent growth model' (LGM; Meredith \& Tisak, 1990), a 'level-only model' (LOM; McArdle \& Aber, 1990) and a 'Markov-simplex model' (MSM; Jöreskog, 1970) were examined. LGM's model longitudinal data is influenced by two latent sources, level and change, and it offers a sound basis for this research. LOM and MSM are two technical alternatives that are tested against LGM to provide a strict test for the use of LGM. Figure 1 summarizes path diagrams of all three models of stability and change, respectively.

In LGMs (see Fig. 1a), the variable $Y_{t}$, which is measured at different occasions, is influenced by two latent sources (level and slope). Inter-individual differences in the initial value are represented by the level factor. Initial values are described by their variance $\left(\psi_{0}\right)$ and their mean $\left(\alpha_{0}\right)$. The second factor represents the change of the variable $Y_{t}$. The factor loadings of the slope factor express the shape of change, which is linear in the example in Fig. 1a. Inter-individual differences in the amount of change are expressed by the variance $\psi_{1}$ of the slope-factor. The aggregated group change is captured by the mean $\left(\alpha_{1}\right)$ of the latent change factor. Model 1A thus assumes that the shape of change is invariant across subjects.

The path diagram in Fig. $1 \mathrm{~b}$ describes a model of stability, the LOM. The repeatedly measured variables $Y_{t}$ are equally influenced by one latent source (level) as indicated by the time-invariant factor loadings. Inter-individual differences on the variable $Y$ are assumed to be stable across the observed time span. Inter-individual differences in true scores are expressed by the variance of the latent variable $\left(\psi_{0}\right)$. Because of the stability of scores, Model 1B assumes that the means of the variables do not differ across occasions. Only one parameter accounts for the observed means $\left(\alpha_{0}\right)$. Associations between the latent variable (level) of Model $1 \mathrm{~B}$ with other variables reflect relations with a stable attribute.

Notions of inter-individual differences in intra-individual change are not useful within a MSM (Fig. 1c). Relations between subsequent measurements of one variable are expressed by the regression coefficients $\beta_{t, t-1}$, which can be interpreted as stability parameters. Individual change is summarized by time-specific (residual) variances $\psi_{\mathrm{t}}$. In contrast to Model 1A, Model $1 \mathrm{C}$ thus does not summarize an individuals' overall change, because the amount of residual change is assumed to vary across occasions (that is, the time-specific variances $\psi_{\mathrm{t}}$ are uncorrelated). Because a simplex model does not assume the existence of a change function that holds true for all individuals in a sample, it is not possible to define inter-individual differences in change within this model. ${ }^{1}$

\footnotetext{
${ }^{\prime} G$ rowth curve models and simplex models may be combined with a hybrid model. We do not report findings based on this
} model because it did not yield an admissible solution. 
The LOM and LGM offer a sound basis for the investigation of inter-individual differences in a stable attribute or a variable changing in a linear fashion, respectively. Therefore, a series of models were fitted separately to the weekly measured action control facets. For each subscale, the best fitting model was selected. The longitudinal models were specified in accordance with the specification provided in Fig. 1, with only one exception; namely, that the residual variances of the observed variables in the LOM and LGM were constrained to be equal across time.
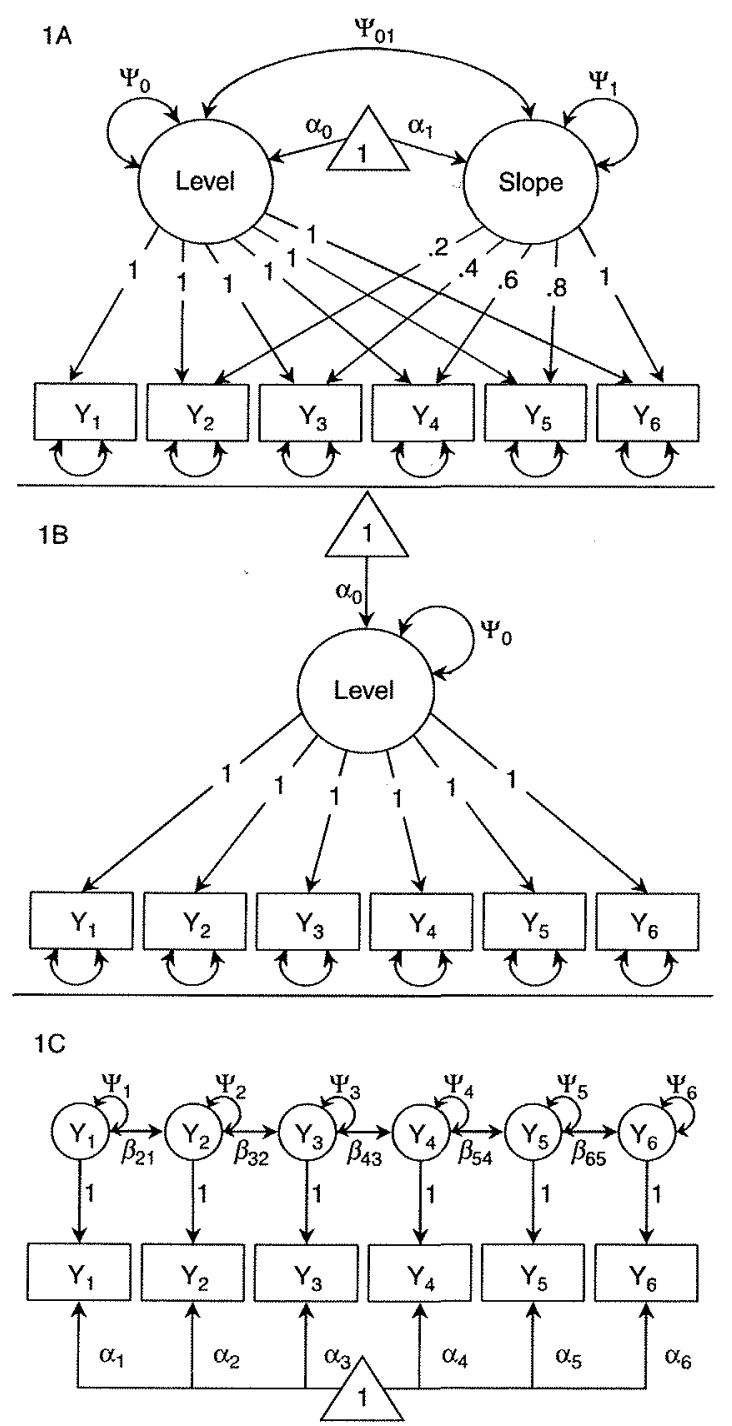

Figure I. Path diagrams representing a 'linear latent growth model' (IA), a 'level-only model' (IB) and a 'Markov-simplex model' (IC). Boxes represent observed variables; circles indicate latent factors. A triangle indicates a constant that defines means or intercepts, respectively. 
Predictive power of the action control facets

Finally, the predictive power of levels and change in action control facets for physical exercise and changes in intentions were tested by hierarchical regression analyses. In order to avoid effects of collinearity, principal component analysis was used beforehand to estimate orthogonal person-specific component scores.

\section{Missing data and variable distributions}

Most longitudinal studies face the problem of missing data. Complete deletion of persons with missing values has been shown to negatively influence the validity of results unless data are missing completely at random. Therefore, missing estimation procedures are preferred over listwise deletion of cases (Little \& Rubin, 1987). In the present study, the problem of missing values was resolved by using direct maximumlikelihood estimation methods in the structural equation modelling approach. All other analyses were conducted after the imputation of missing values with the expectation maximization procedure (Enders, 2001). All structural equation models (SEM; e.g. Bollen, 1989) were fitted with the programme Mplus 3 (Muthén \& Muthén, 1998-2004). Model fit was assessed by the $\chi^{2}$ statistic, the Tucker-Lewis Index (TLD, and the root mean square error of approximation (RMSEA). In the presence of non-normally distributed data, fit indices and standard errors of the model parameters are biased under the usually employed maximum-likelihood estimation procedure. Because the longitudinal measures deviate from a normal distribution, all subsequent structural equation models were fitted by means of a maximum-likelihood estimator that is robust against violations of the normal distribution. Furthermore, the robust maximumlikelihood estimator employed (MLR; Yuan \& Bentler, 2000) is also capable of handling the missing data problem. MLR provides direct estimates of model parameters that are based on all available data points.

\section{Results}

\section{Stability and variability of the three action control facets}

Five multi-level confirmatory factor analytic models with a different number of factors on the between-and within-person level were fitted to the longitudinal data. Fit statistics for these models are given in Table 2.

A model that assumes a one-dimensional structure at both levels (Model 1) did not fit the data. The second model, assuming a three-factor structure at the within level and

Table 2. Goodness-of-fit indices for the multi-level confirmatory factor analysis models fitted to the weekly measured action control items

\begin{tabular}{|c|c|c|c|c|}
\hline & & $\chi^{2}(d f)$ & TLI & RMSEA \\
\hline Model I & $|F(B)+| F(W)$ & $106.44(18)$ & .772 & .085 \\
\hline Model 2 & $I F(B)+3 F(W)$ & $51.14(15)$ & .888 & .059 \\
\hline Model 3 & $3 F(B)+1 F(W)$ & $67.65(15)$ & .837 & .071 \\
\hline Model 4 & $3 F(B)+3 F(W)$ & $22.72(12)$ & .959 & .036 \\
\hline Model 5 & $3 F(B)+3 F(W)+$ Loading Invariance & $24.94(15)$ & .969 & .031 \\
\hline
\end{tabular}

Note. F: factors; (B): between-individual level; (W): within-individual level. 
one-factor at the between level provided a better fit as compared with the first model $\left(\Delta \chi_{3}^{2}=41.94, p<.01\right)^{2}$ A model specifying a three-dimensional structure at the between level and a one-factor at the within level (Model $3 ; \Delta \chi_{3}^{2}=56.80, p<.01$ ) showed an improved fit. Model 4, specifying a three-factor structure at both levels provided a far better fit to the data as compared with Model $2\left(\Delta \chi_{3}^{2}=44.55, p<.01\right)$ and to Model $3\left(\Delta \chi_{3}^{2}=36.64, p<.01\right)$, respectively. In order to compare the amount of inter-individual variability with the amount of intra-individual variability, the factor loadings of the between-person level were constrained to be the same as those at the within-person level. This final Model 5 (see Fig. 2) exhibited virtually the same fit to the data as Model $4\left(\Delta \chi_{3}^{2}=3.19, p>.25\right)$, and even outperformed Model 4 in terms of TLI and RMSEA indices.

For the purpose of comparing the relative amount of intra-individual variability to stable inter-individual differences (e.g. Nesselroade \& Salthouse, 2004), we computed the ratio of within-person construct variability to between-person construct variability (WBV). In situations where a construct does not exhibit any within-individual fluctuations, WBV takes a value of 0 . A value of $W B V=1$ represents the case where the intra-individual variance equals the between-person variability. Values of WBV $>1$ are indicative for the dominance of within-person fluctuations as compared with stable inter-individual differences. The WBV can thus be interpreted as an index of the relative importance of intra-individual fluctuations opposed to stable differences between persons.

The lowest ratio of variance terms was determined for the awareness of standards $(\mathrm{WBV}=.48)$, indicating that this facet is mainly dominated by a stable component of inter-individual differences. Higher relative week-to-week variation was found for selfregulatory effort $(\mathrm{WBV}=.65)$ and for self-monitoring (WBV $=.85)$. The facet selfmonitoring, thus, exhibits roughly the same amount of stable inter-individual differences as compared with the amount of week-to-week fluctuation.

MCFA indicated that the three facets were highly intercorrelated at the between- as well as at the within-person level. Self-regulatory effort and self-monitoring exhibited the highest correlations at both levels of analysis (between: $r=.94, p<.01$; within: $r=.88, p<.01$ ), indicating a strong overlap between these two facets. Taken together, the MCFA analyses corroborated the expected three-factor structure of action control. However, as indicated by the high construct correlations at both levels of analysis, the facets self-regulatory effort and self-monitoring seem to be somewhat redundant. These facets could thus be aggregated into a single score without major loss of information. However, an aggregation of scale scores may be problematic, because the measures differed in their time-specific components, as indicated by their different WBVs. Therefore, the change of both facets was investigated once more subsequently.

Type of change in the action control facets

The within-individual variability terms in the MCFA models cannot resolve the questions about the type of change and inter-individual differences in the amount of individual change. Different longitudinal models were fitted to the data in order to investigate the

\footnotetext{
${ }^{2}$ The $\chi^{2}$ difference tests under MLR estimation do not correspond to the row differences in the $\chi^{2}$ yolues of two competing models because these differences do not follow a $\chi^{2}$ distribution.
} 

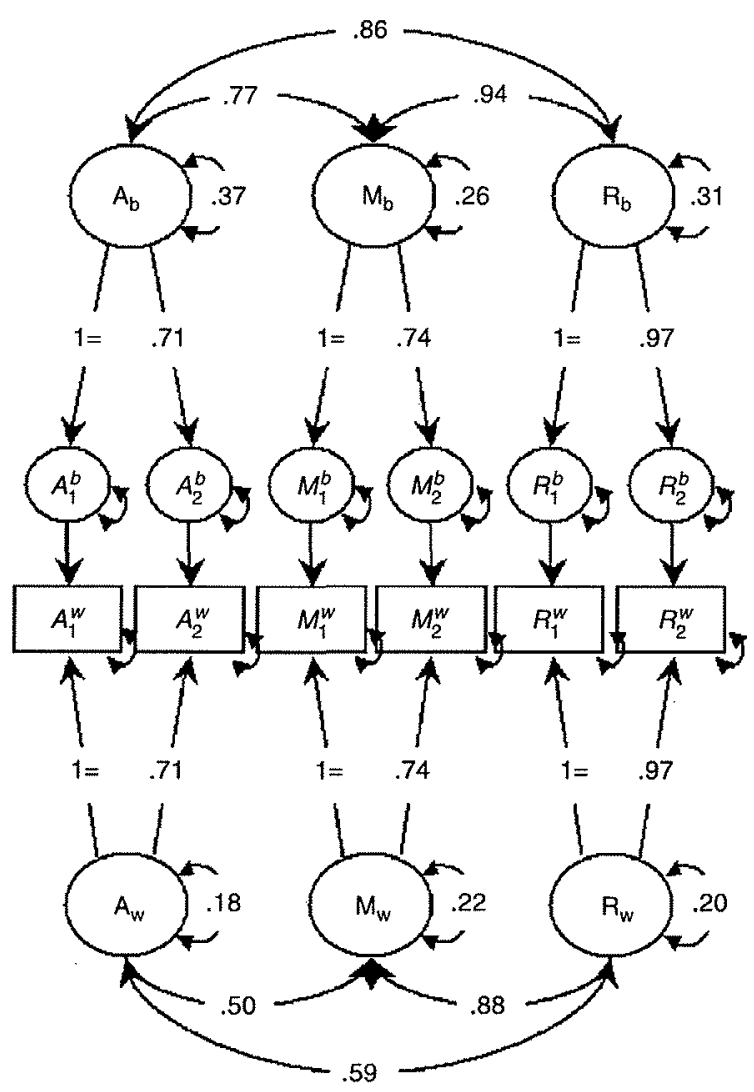

Figure 2. Path diagram for the final multi-level confirmatory factor model. The upper part of the figure represents the between-individuals (b) structure of action control. The lower part represents the structure of weekly fluctuation in action control $(w)$. Factor loadings and factor variances are reported in an unstandardized metric. Association between factors are given as correlation coefficients. A: awareness of standards; $M$ : self-monitoring; $R$ : self-regulatory effort; $=$ : parameter fixed for identification purposes.

type of change in the action control facets across the six-week period. The suitability of a LOM, a LGM with a linear change function, and a MSM was scrutinized.

Awareness of standards consists mainly of a stable variance component, as can be seen in Table 3. The LOM fits the longitudinal data very well, and the fit cannot be improved by adding a linear change component in the LGM $\left(\Delta \chi_{3}^{2}=2.83, p>.25\right)$. Thus, stability seems to be a reasonable assumption for this scale. Additionally, a MSM failed to fit the data better. ${ }^{3}$ The finding that awareness of standards is dominated by a stable component is in accordance with the findings based on the MCFA.

The fit indices for self-monitoring indicated a linear change component. The inclusion of a linear change factor in the LGM significantly improved the model fit

${ }^{3}$ The MSM cannot be compored directly to an LOM or an LGM via o $\chi^{2}$ difference test because these models are not nested. Model comparison must rely on the mere comparison of the fit indices employed. 
Table 3. Goodness-of-fit indices for the models fitted to the weekly measured action control scales

\begin{tabular}{|c|c|c|c|c|c|c|c|c|c|}
\hline & \multicolumn{3}{|c|}{ Awareness of standards } & \multicolumn{3}{|c|}{ Self-monitoring } & \multicolumn{3}{|c|}{ Self-regulatory effort } \\
\hline & $x^{2}(d f)$ & $T L I$ & RMSEA & $x^{2}(d f)$ & $T U$ & RMSEA & $x^{2}(d f)$ & $T L I$ & RMSEA \\
\hline LOM & $39.86(24)$ & .951 & .074 & $62.49(24)$ & .873 & .115 & $38.28(24)$ & .941 & .070 \\
\hline LGM & $36.54(21)$ & .945 & .078 & $34.40(2 \mathrm{I})$ & .950 & .072 & $28.39(21)$ & .965 & .054 \\
\hline MSM & $71.37(10)$ & .545 & .225 & $25.55(10)$ & .877 & .113 & $48.54(10)$ & .620 & .179 \\
\hline
\end{tabular}

Note: LOM: level-only model; LGM: linear change model; MSM: Markov-simplex model.

compared with a $\operatorname{LOM}\left(\Delta \chi_{3}^{2}=37.44, p<.01\right)$. Furthermore, the LGM provided a better representation of the data as compared with the MSM: the LGM attained more favourable TLI and RMSEA indices and it was more parsimonious. The results for selfregulatory effort were similar to those of self-monitoring. The LGM was superior to LOM $\left(\Delta \chi_{3}^{2}=10.16, p<.05\right)$. Moreover, a MSM did not fit the data. The structural parameter of the final models for the action control components are summarized in Table 4.

Table 4. Structural parameters of the final models of the action control scales

\begin{tabular}{lccc}
\hline & Awareness of standards & Self-monitoring & Self-regulatory effort \\
\hline Mean initial value & $3.32^{* *}$ & $3.23^{* *}$ & $3.34^{* *}$ \\
Mean linear change & - & .07 & -.01 \\
Variance initial value & $.32 *$ & $.48^{* *}$ & $.32^{* *}$ \\
Variance linear change & - & $.41^{* *}$ & $.23^{*}$ \\
Factor correlation & - & $-.50^{* *}$ & -.18 \\
\hline
\end{tabular}

Note: $* p<.05 ; * p<.01$.

As can be seen in Table 4 , all action control facets exhibit comparable initial values. The facets that entail a change component (self-monitoring and self-regulatory effort) exhibit a non-significant mean change, indicating that the sample did not show a systematic mean change over time. However, as indicated by the significant variance of the linear components, individuals differ in their amount of linear increases and decreases on the self-monitoring and self-regulatory effort scales, respectively. For these measures, however, positive and negative directions of change compensate each other. Taken together, the findings based on the longitudinal models were in accordance with those provided by the MCFAs. The constructs with the highest intra-individual variance in the MCFAs demonstrated the highest variance in the linear change components in the LGM models.

In sum, the analyses of the longitudinal properties of the action control facets offered the possibility of further investigating the predictive power of inter-individual differences in the initial levels of the action control facets as well as in intra-individual changes in self-monitoring and self-regulatory effort for changes in intentions and physical exercise. In the case of the scale awareness of standards, only the level can be analysed. 


\section{Relations between stability and change in the action control facets}

Sample size did not allow the estimation of multivariate linear growth models employing SEM. Therefore, a 2-stage approach was chosen to analyse the relation between the stable and dynamic components of the different variables. The 2-stage strategy involved a regression-based estimation of individuals' values on initial level and amount of linear change in the first place. Second, the derived individual estimates were related to one another as well as to different outcomes.

The SEM analyses had revealed that awareness of standards could best be described as stable. Thus, in the subsequent analyses, the six weekly scores of this scale were averaged. For self-monitoring and self-regulatory effort, the individuals' amount of linear change was estimated by a simple regression procedure. The individuals' scores on the repeatedly measured variables were regressed separately on a linear time contrast $[0.2$ .4 .6 .81 ] . Regression models were thus fitted for each person separately. A person's regression intercept represented her or his initial value, while the person-specific regression slope represented a measure of her or his amount of linear change. The correlations of the estimated components are presented in Table 5.

Table 5. Correlations of the regression-based estimates of the initial values (I) and linear change components (L) of the action control subscales

\begin{tabular}{lllllll}
\hline & & {$[1]$} & {$[2]$} & {$[3]$} & {$[4]$} & {$[5]$} \\
\hline I Awareness of standards & {$[1]$} & 1 & & & & \\
I Self-monitoring & {$[2]$} & $.32^{* *}$ & 1 & & & \\
I Self-regulatory effort & {$[3]$} & $.57^{* *}$ & $.64^{* *}$ & 1 & & \\
L Self-monitoring & {$[4]$} & $.23^{*}$ & $-.61^{* *}$ & $-.29 * *$ & 1 & \\
L Self-regulatory effort & {$[5]$} & .14 & $-.28^{* *}$ & $-.49^{* *}$ & $.68^{* *}$ & 1 \\
\hline
\end{tabular}

Note: $* p<.05 ; * p<.01$.

The overall measure of awareness of standards correlated positively with the initial values of the other subscales and also correlated with their linear change components. The change in self-monitoring and self-regulatory effort seemed to develop in a parallel fashion. Their initial values were correlated substantially $(r=.64, p<.01)$, as well as their linear change components $(r=.68, p<.01)$. The pronounced correlations between the initial values and change components of both facets agree with the previous findings, based on the MCFA models. However, the correlations reported here are likely to be underestimated because of the method of estimating the initial values and linear change components. The high correlations between the initial values and the change in the self-regulatory effort and self-monitoring facets as well as the high correlations determined by the MCFA suggest an aggregation of the initial values and change components, respectively, of these two facets.

A principal component analysis (PCA) with varimax rotation of the initial values and change-scores of the self-monitoring and self-regulatory effort scales was conducted to estimate person-specific component scores. It suggested the extraction of two components ( $83.2 \%$ explained variance) that can clearly be interpreted as an initial level factor ( $42 \%$ explained variance) and as a change factor ( $41.2 \%$ explained variance). Both factors were characterized by substantial positive primary loadings $(>.86)$ and by minor negative secondary loadings $(-.17$ to -.28$)$. The results of this PCA allowed estimating 
individual component scores for the initial level component and for the linear change component of self-monitoring and self-regulatory effort together. These scores were used in subsequent regression analyses. The use of these scores circumvents problems associated with suppression effects in multiple regressions. Furthermore, the component scores are more reliable as compared with the raw scores.

\section{Predictive power of the action control facets}

Two hierarchical regression analyses were performed to predict Time 2 intentions and Time 2 recommended strenuous exercise, respectively. The first analysis addressed the question of whether the action control facets predict changes in behavioural intentions. In the first step, behavioural intentions at Time 1 were entered. In the second step, the person-specific initial values for awareness of standards and the level component of selfmonitoring and self-regulatory effort were entered into the equation. In the third step, the linear change component of self-monitoring and self-regulatory effort were added to the prediction model to test whether the change contributed to the prediction over and above the level scores.

Behavioural intentions exhibited high retest stability $(\beta=0.57, p<.01)$. However, the level components in Step 2 improved the prediction $\left(\Delta R^{2}=.13, p<.01\right)$. The inclusion of the action-control level variables reduced the effect of behavioural intentions at Time 1 . Since intentions at Time 1 were significantly related to the level component of self-monitoring and self-regulatory effort $(r=.38, p<.01)$ and to the awareness of standards $(r=.39, p<.01)$, the effect of intentions at Time 1 on intentions at Time 2 was partly mediated by the initial levels of action control. Entering the change component of self-monitoring and self-regulatory effort in the third step did not change the effect of Time 1 behavioural intentions. Sobel tests for the indirect effects based on the final model confirmed that only the level component of selfmonitoring and awareness of standards mediated the relationship between intentions at Time 1 and Time $2\left(z_{\text {sobel }}=3.05, p<.01\right)$, whereas awareness of standards and the change component did not. However, the change component further improved the prediction of Time 2 behavioural intentions $\left(\Delta R^{2}=.05, p<.01\right)$. Both component scores, initial level and linear change, were significantly related to Time 2 behavioural intentions. In sum, they accounted for more variance in intentions two months after discharge than the baseline-measure of intentions (see Table 6).

The second hierarchical regression analysis was performed with recommended strenuous exercise as the dependent variable. Here, the same order of steps was applied, and in the last step, behavioural intentions at Time 2 were entered to the regression equation. ${ }^{4}$ The first step revealed that Time 1 behavioural intentions exerted a small direct effect $(\beta=0.19, p<.05)$ on recommended strenuous exercise. In the second step, the level components of the action control facets were entered and added significantly to the prediction of exercise $\left(\Delta R^{2}=.07, p<.01\right)$. After including the level variables of action control, the effect of Time 1 intentions was no longer significant. Furthermore, only the level component of self-monitoring and self-regulatory effort was a significant predictor, whereas awareness of standards was not significant. In the third step, the change component of self-monitoring and self-regulatory effort further

\footnotetext{
${ }^{4}$ Interactions omong action control components as well as interactions between intentions and action control components in
} predicting intentions and behaviour were tested. None of these interactions was significant. 
Table 6. Multiple regressions (standardized coefficients) predicting intentions and recommended strenuous exercise 2 months after discharge from rehabilitation

\begin{tabular}{|c|c|c|c|c|c|c|c|}
\hline & \multicolumn{3}{|c|}{ Intentions (Time 2) } & \multicolumn{4}{|c|}{ Exercise (Time 2) } \\
\hline & Step 1 & Step 2 & Step 3 & Step 1 & Step 2 & Step 3 & Step 4 \\
\hline Intention (Time I) & $.57 * *$ & $.40 * *$ & $.40 \%$ & $.19 *$ & .07 & .07 & .00 \\
\hline Awareness of standards & & $.17^{*}$ & .03 & & .07 & -.11 & -.12 \\
\hline Initial level (Comp.) & & $.27 * *$ & $.36 * *$ & & $.23 *$ & $.35 \%$ & $.29 *$ \\
\hline Linear change (Comp.) & & & $.25^{* \alpha}$ & & & $.31 * *$ & $.27 \%$ \\
\hline Intention (Time 2) & & & & & & & .17 \\
\hline$R^{2}$ & .33 & .45 & .49 & .04 & .10 & .18 & .19 \\
\hline
\end{tabular}

Note: Initial level: principal component defined by the initial level of self-monitoring and self-regulatory effort; linear change: principal component defined by the linear change of self-monitoring and selfregulatory effort.

$* p<.05 ; * p<.01$.

enhanced the predictive power $\left(\Delta R^{2}=.08, p<.01\right)$. Sobel tests for the indirect effects indicated that the intention-behaviour relationship was mediated by the initial level of self-monitoring and regulatory effort $\left(z_{\text {Sobel }}=2.53, p=.01\right)$ and not by the change component or by awareness of standards.

Finally, the inclusion of intentions at Time 2 , which was assessed simultaneously to the behavioural outcome, did not change the pattern of findings, although the bivariate correlation between intentions at Time 2 and recommended strenuous exercise at Time 2 was significant $(r=.34, p<.01)$.

In sum, the scores of the level and the linear change component of self-monitoring and self-regulatory effort were the only significant predictors of recommended strenuous exercise two months after discharge. The effect of Time 1 intentions was mediated by the level component of the action control facets self-monitoring and selfregulatory effort. Intentions measured at Time 2 could not add any prediction of variance in recommended strenuous exercise over and above the effects of the different components of the action-control facets.

\section{Discussion}

The present study has examined the longitudinal relationships and predictive power of levels and change in three facets of action control: self-monitoring, awareness of standards and self-regulatory effort over six weeks. The longitudinal factor structure of the six action control items was tested first, confirming three dimensions of action control. This analysis confirmed that it is sensible to distinguish between the three facets because they differed in both the differences between subjects as well as their week-toweek fluctuation within subjects. This implies that inter-individual and intra-individual differences are best accounted for by the three factors self-monitoring, awareness of standards and self-regulatory effort.

Two of these dimensions, self-monitoring and self-regulatory effort, changed over time in a linear fashion. Thus, people seem to differ in their absolute level of action control as well as in their amount of change within the first 6 weeks after discharge from rehabilitation. In contrast to self-monitoring and self-regulatory effort, the third facet, 
awareness of standards, was characterized by stability. Over 6 weeks, individuals maintained stable levels of awareness of standards. This stability might be due to the rehabilitation treatment in which the standards were defined and trained. However, implementing the physical exercise intention into action requires self-monitoring and effort that had not constituted therapy goals of the traditional rehabilitation treatment.

These initial findings provided a sound basis for the subsequent analysis of initial level and change of action control facets in terms of their predictive power for intention and physical exercise changes. Based on the multivariate analyses, the component scores for level and change of self-monitoring and effort as well as the mean of awareness of standards served as predictors in regression analyses. For these final analyses, individual estimates for initial level and change in self-monitoring and selfregulatory effort derived from the two LGMs were combined because these individual scores were highly interrelated.

Level and change in action control predicted changes in bebavioural intentions within the first 2 months after discharge. The level component of self-monitoring and regulatory effort mediated the relationship between intentions at Time 1 and Time 2. The action control measures substantially improved the prediction in Time 2 intention over and above the baseline measure. These findings contribute to the discussion of intention stability as a proxy of volitional processes (Bagozzi \& Yi, 1989; Conner et al., 2000 ). Changes in intentions can be attributed to both lower levels of and a decline in action control. Studying action control allows a better understanding of intention stability and contributes to the identification of particular mechanisms underlying instability in intentions.

Regression analyses showed that both levels and changes of action control were predictive for physical exercise and that the influence of intentions at baseline is fully mediated by the initial level of self-monitoring and self-regulatory effort. This is in line with previous findings (Sniehotta et al., 2005). Even the follow-up measure of behavioural intentions, which was assessed about 3 weeks after the last weekly questionnaire and at the same point in time as the physical exercise outcome, did not contribute to the prediction over and above action control. This underlines the central role of action control in the self-regulation of behaviour.

Awareness of standards did not contribute to any of these regressions. This does not necessarily imply that this variable has no predictive power at all. It is significantly correlated with the outcomes $(r=.50, p<.01$ with intentions at Time $2, r=.34$, $p<.01$ with exercise at Time 2). The other measures may have outperformed it because essentially they entail the same information (see Table 5). Standards seem to be indispensable in action control. Given the high mean value in the present study, individual differences may have been underestimated. The weekly questionnaires may have contributed to relatively high levels of awareness of standards in the sample. Furthermore, it was not the aim of the analyses to test the facets against each other. The regression analyses pursued mainly the goal to determine whether changes in action control were related to subsequent outcomes, and this was strongly supported by the data.

The idea of feedback control implies that the action control facets cannot operate independently. Rather, they should be regarded as components of one functional process. Our analyses revealed support for the assumed factor structure, but at the same time, the findings indicated substantial interrelations between the three factors. Depending on the research question, it thus seems reasonable to use either an aggregate score or separate measures for the action control facets, respectively. Within studies in 
which the action-control construct is applied only at one point in time, it would be preferable to use an aggregate score, while studies that aim at the investigation of longitudinal dynamics in action control should rather rely on facet scores as was done here.

Participants in this study had high overall levels in awareness of standards, selfmonitoring and self-regulatory effort. This could be partly the result of participating in the study and receiving weekly questionnaires and partly the result of the rehabilitation treatment they had undergone. Higher levels in all three facets of action control corresponded positively with changes in intentions. Individuals with high action control maintained or even increased their behavioural intention levels, which were already high in this particular sample of cardiac patients. It can be assumed that awareness of standards and the ongoing effort in monitoring and control have reinforced the intentions. Likewise, high levels in action control lead to higher levels in recommended physical exercise. Given the high intentions to do so, action control enabled participants to act on their intentions. The facilitating role of negative feedback in intended behaviour change has been addressed by several authors (Baumeister et al., 1994; Carver \& Scheier, 1998). This study provides empirical evidence in a unique natural setting where failures of self-regulatory attempts can have life-threatening consequences.

Changes in self-monitoring and self-regulatory effort appeared to be linear. While changes in both components are negatively correlated with their initial values, changes in self-monitoring are positively associated with awareness of standards. Changes in action control cognitions reflect the experience of improvement or worsening in control administered, and this may include possible lapses in the course of behaviour change (Baumeister et al, 1994). Consequently, individuals who experience a decline are more likely to lower their intentions or fail to act on them.

The action control items are formulated in correspondence to the behavioural outcome and tailored to the time lags under study. This measure differs from trait measures of self-regulation and action control (e.g. Kuhl \& Fuhrmann, 1998; Orbell, 2003). A trait perspective of action control processes is well in line with theory and previous findings (e.g. Mischel, Shoda, \& Rodriguez, 1989; Orbell, 2003; Schroder \& Schwarzer, 2004). However, the present study provides evidence that changes in behaviour-specific action control can account for behaviour change, especially in early stages. Trait measures of volition, however, may correspond to broader outcomes and may be less applicable if particular outcomes, such as physical exercise, are examined. The principle of correspondence between behavioural intentions and behavioural measures (Ajzen, 1991; Courneya, 1994) can easily be extended and applied to volitional measures of negative feedback control. Instead of time- and contentunspecific measures, the action control facets of awareness of standards, self-monitoring and self-regulatory effort can be assessed in correspondence to the target behaviour, its context and the timeline. In a study on long-term changes in physical exercise after rehabilitation, a specific measure of action control was shown to mediate fully the influence of behavioural intentions and to mediate partly the influence of other more distal volitional measures, such as action planning and volitional self-efficacy (Snichotta et al.,2005). Thus, there is good reason to amplify existing measures by the present one.

Some results must be interpreted with caution, however. Firstly, the sample analysed is relatively small, and the statistical power for detecting a significant change variance may be reduced. The analytic procedures used in this research account for this by using conservative methods and by carefully exploring the assumptions for the final analyses. Secondly, the data represent only a relatively short time span following the core idea of 
this research; namely, that these specific 6 weeks may be the crucial ones. Future research may also incorporate longer time intervals to investigate whether change factors, present in the first weeks, differ from those at later time periods. Thirdly, the measure of behavioural intentions used in this study covers a slightly broader range of behaviour recommended to cardiac patients than the outcome measure ('recommended strenuous exercise'). This measure has been validated in previous research (Sniehotta et al., in press). Although the 6 items composing the intentions scale are homogeneous $(\alpha=82)$, the lack of correspondence might have led to an underestimation of the effect of intentions, which is a limitation of this study. The effect of intentions on behaviour is indeed lower than those in previous meta-analyses (e.g. Armitage \& Conner, 2001). The particular intention-physical exercise relationship in cardiac patients, however, is a weak one, as documented by other studies Johnston, Johnston, Pollard, Kinmonth, \& Mant, 2004). The findings that levels and change in action control facets were highly predictive of both physical exercise and changes in intentions indicate that is the level of correspondence between the measures was sufficient to study their interrelations over time. However, future research is needed to confirm whether action control fully mediates the effect of intentions on behaviour when intentions are assessed in strict correspondence to the behavioural outcome measure.

An unresolved issue in the present study is the concurrent validity of action control compared with other established constructs in health behaviour change, such as PBC (Ajzen, 1991) or perceived self-efficacy (Bandura, 1997). Both PBC and self-efficacy are prospective cognitions about control that are likely to be related to action control. The aim of the present study was to introduce the three facets of action control, awareness of standards, self-monitoring and regulatory effort and to examine whether they predict changes in behaviour and intentions. Subsequent studies should address the validity of the action control facets in comparison to PBC and self-efficacy.

This study has shed more light on the self-regulatory mechanisms that may operate after a behavioural intention has been formed. Three facets of action control have proven useful to establish more proximal antecedents of behaviour change as compared with the common prediction models that focus mainly on intentions. The action control construct, measured in the suggested three-dimensional manner, may augment the existing social cognition models to overcome the intention-behaviour gap. Based on this theoretical conception, behaviour change interventions can be formulated and the effects of these interventions can be mediated using the measure introduced in this research (Sniehotta, Scholz, Schwarzer, Fuhrmann, Kiwus \& Völler, 2005)

\section{Acknowledgements}

We would like to thank Derek Johnston and Paschal Sheeran for helpful comments on an earlier draft of this paper.

\section{References}

Abraham, C., Sheeran, P., \& Johnston, M. (1998). From health beliefs to self-regulation: Theoretical advances in the psychology of action control. Psychology and Health, 13, 569-591.

Ajzen, I. (1991). The theory of planned behavior. Organisational Bebavior and Human Decision Processes, 50, 179-211. 
Armitage, C. J., \& Conner, M. (2001). Efficacy of the theory of planned behaviour: A meta-analytic review. British Journal of Social Psycbology, 40, 471-495.

Bagozzi, R. P., \& Yi, Y. (1989). The degree of formation as a moderator of the attitude-behavior relation. Social Psychology Quarterly, 52, 913-929.

Bandura, A. (1997). Self-efficacy: The exercise of control. New York: Freeman.

Bandura, A., \& Cervone, D. (1983). Self-evaluative and self-efficacy mechanisms governing the motivational effects of goal systems. Journal of Personality and Social Psychology, 45, 1017-1028.

Baumeister, R. F, Heatherton, T. F, \& Tìce, D. (1994). Losing control: How and why people fail at self-regulation. San Diego, CA: Academic Press.

Bollen, K. A. (1989). Structural equations with latent variables. New York: Wiley.

Carver, C. S., \& Scheier, M. F (1998). On the self-regulation of bebavior. New York: Cambridge University Press.

Conner, M., Sheeran, P., Norman, P., \& Armitage, C. J. (2000). Temporal stability as a moderator of relationships in the theory of planned behaviour. British Journal of Social Psychology, 39, 469-493.

Courneya, K. S. (1994). Predicting repeated behavior from intention: The issue of scale correspondence. Journal of Applied Social Psychology, 24, 580-594.

Einstein, G. O., McDaniel, M. A., Willifort, C. L., Pagan, J. L., \& Dismukes, R. K. (2003). Forgetting of intentions in demanding situations is rapid. Journal of Experimental Psycbology: Applied, 9 , 147-162.

Enders, C. K. (2001). A primer on maximum likelihood algorithms available for use with missing data. Structural Equation Modeling, 8, 128-141.

Fishbein, M., \& Ajzen, I. (1975). Belief, attitude, intention and bebavior: An introduction to theory and research. Reading, MA: Addison-Wesley.

Ford, D. H. (1987). Humans as self-constructing living systems. A developmental perspective on bebavior and personality. Hillsdale, NJ: Erlbaum.

Gollwitzer, P. M. (1999). Implementation intentions. Strong effects of simple plans. American Psychologist, 54, 493-503.

Hox, J. (2002). Multi-level analysis: Techniques and applications. Mahwah, NJ: Erlbaum.

Johnston, D. W., Johnston, M., Pollard, B., Kinmonth, A. L., \& Mant, D. (2004). Motivation is not enough: Prediction of risk behavior following diagnosis of coronary heart disease from the theory of planned behavior. Healtb Psycbology, 23, 533-538.

Jöreskog, K. G. (1970). Estimation and testing simplex models. Britisb Joumal of Mathematical and Statistical Psychology, 23, 121-145.

Kanfer, F. H., \& Karoly, P. (1972). Self-control: A behavioristic excursion into the lion's den. Bebavioral Therapy, 3, 398-416.

Karoly, P. (1993). Mechanisms of self-regulation: A systems view, Annual Review of Psychology, $44,23-52$

Kuhl, J., \& Fuhrmann, A. (1998). Decomposing self-regulation and self-control: The volitional components checklist. In J. Heckhausen \& C. Dreck (Eds.), Life-span perspective on motivation and control (pp. 19-45). Mahwah, NJ: Erlbaum.

Kuhl, J., \& Goschke, T. (1994). State orientation and the activation and retrieval of intentions from memory. In J. Kuhl \& J. Beckmann (Eds.), Volition and personality: Action versus state orientation (pp. 127-154). Göttingen, Germany/Toronto: Hogrefe.

Kuhl, J., \& Kazén-Saad, M. (1989). Volition and self-regulation: Memory mechanisms mediating the maintenance of intentions. In W. A. Hersberger (Ed.), Volitional action (pp. 387-407). Dordrecht, The Netherlands: Nijhoff.

Lippke, S., Ziegelmann, J. P., \& Schwarzer, R. (2004). Behavioral intentions and action plans promote physical exercise: A longitudinal study with orthopedic rehabilitation patients. Journal of Sport and Exercise Psychology, 26, 470-483.

Little, R. J. A., \& Rubin, D. B. (1987). Statistical analysis with missing data. New York: Wiley. 
Maes, S., \& Karoly, P. (2005). Self-regulation assessment and intervention in physical health and illness: A review. Applied Psychology: An International Review, 54, 267-299.

McArdle, J. J., \& Aber, M. S. (1990). Patterns of change within latent structural equation models. In A. von Eye (Ed.), Statistical methods in longitudinal research: Principles and methods of structuring change (pp. 151-223). New York: Academic Press.

McDaniel, M. A., \& Einstein, G.O. (2000). Strategic and automatic processes in prospective memory retrieval: A multiprocess framework. Applied Cognitive Psycbology, 14, \$127-\$144. Meredith, W., \& Tisak, J. (1990). Latent curve analysis, Psycbometrika, 55, 107-122.

Mischel, W., Shoda, Y., \& Rodriguez, M. I. (1989). Delay of gratification in children. Science, 244, 933-938.

Muthén, L. K., \& Muthén, B. O. (1998-2004). Mplus user's guide (3rd ed). Los Angeles, CA: Muthén and Muthén.

Nesselroade, J. R., \& Salthouse, T. A. (2004). Methodological and theoretical implications of intraindividual variability in perceptual motor performance. Joumals of Gerontology: Psychological Sciences, 59, 49-55.

Orbell, S. (2003). Personality systems interactions theory and the theory of planned behaviour: Evidence that self-regulatory volitional components enhance enactment of studying behaviour. British Journal of Social Psychology, 42, 95-112.

Orbell, S., \& Sheeran, P. (1998). Inclined abstainers: A problem for predicting health behaviour. Britisb Joumal of Social Psychology, 37, 151-166.

Schroder, K. E. E., \& Schwarzer, R. (2004). Habitual self-control and the management of health behaviour amongst heart patients. Social Science and Medicine, 60, 859-875.

Sheeran, P. (2002). Intention-behaviour relations: A conceptual and empirical review. In $M$ Hewstone \& W. Stroebe (Eds.), European review of social psychology, (Vol. 12, pp. 1-36). Chichester: Wiley.

Sheeran, P., Trafimow, D., \& Armitage, C. J. (2003). Predicting behaviour from perceived behavioural control: Tests of the accuracy assumption of the theory of planned behaviour. British Journal of Social Psychology, 42, 393-410.

Sniehotta, F. F, Scholz, U., \& Schwarzer, R. (2005). Bridging the intention-behaviour gap: Planning, self-efficacy, and action control in the adoption and maintenance of physical exercise. Psychology and Health, 20, 143-160.

Sniehotta, F. F., Schwarzer, R., Scholz, U., \& Schüz, B. (2005). Action planning and coping planning for long-term lifestyle change: Theory and assessment. European fournal of Social Psycbology, 35, 565-576.

Sniehotta, F. F, Scholz, U., Schwarzer, R., Fuhrmann, B., Kiwus, U., \& Völler, H. (2005). Long-term effects of two psychological interventions on physical exercise and self-regulation following coronary rehabilitation. International Joumal of Bebavional Medicine, 12, 244-255.

Sutton, S. R. (2001). Psychosocial theories of health behavior. In N. J. Smelser \& P. B. Baltes (Eds.), The international encyclopedia of social and bebavioural sciences (pp. 6499-6506). Oxford: Elsevier.

Yuan, K. H., \& Bentler, P. M. (2000). Three likelihood-based methods for mean and covariance structure analysis with nonnormal missing data. In M. E. Sobel \& M. P. Becker (Eds.), Sociological methodology 2000 (pp. 165-200). Washington, DC: ASA. 